GA-A24257

\title{
ELECTRON CYCLOTRON CURRENT DRIVE EFFICIENCY IN GENERAL TOKAMAK GEOMETRY
}

\author{
by \\ Y.R. LIN-LIU, V.S. CHAN, and R. PRATER
}

MARCH 2003 
DISCLAIMER

This report was prepared as an account of work sponsored by an agency of the United States Government. Neither the United States Government nor any agency thereof, nor any of their employees, makes any warranty, express or implied, or assumes any legal liability or responsibility for the accuracy, completeness, or usefulness of any information, apparatus, product, or process disclosed, or represents that its use would not infringe privately owned rights. Reference herein to any specific commercial product, process, or service by trade name, trademark, manufacturer, or otherwise, does not necessarily constitute or imply its endorsement, recommendation, or favoring by the United States Government or any agency thereof. The views and opinions of authors expressed herein do not necessarily state or reflect those of the United States Government or any agency thereof. 
GA-A24257

\title{
ELECTRON CYCLOTRON CURRENT DRIVE EFFICIENCY IN GENERAL TOKAMAK GEOMETRY
}

\author{
Y.R. LIN-LIU, V.S. CHAN, and R. PRATER
}

This is a preprint of a paper to be submitted for publication in Phys. Plasmas.

\author{
Work supported in part by \\ the U.S. Department of Energy under \\ DE-AC03-99ER54463 and DE-FG03-95ER54309
}




\begin{abstract}
Green's-function techniques are used to calculate electron cyclotron current drive (ECCD) efficiency in general tokamak geometry in the low-collisionality regime. Fully relativistic electron dynamics is employed in the theoretical formulation. The highvelocity collision model is used to model Coulomb collisions and a simplified quasilinear rf diffusion operator describes wave-particle interactions. The approximate analytic solutions which are benchmarked with a widely used ECCD model, facilitate time-dependent simulations of tokamak operational scenarios using the non-inductive current drive of electron cyclotron waves.
\end{abstract}




\section{INTRODUCTION}

Electron cyclotron current drive (ECCD) is the generation of electrical currents in plasmas by the application of electromagnetic waves at frequencies near the electron cyclotron frequency or its low harmonics. Seminal discussions of the physics of ECCD were first made by Ohkawa ${ }^{1}$ and Fisch and Boozer ${ }^{2}$. Subsequent theoretical investigations $^{3-12}$ of ECCD were mostly specific to tokamak geometry and elaborated on the reduction of the current drive efficiency which originates from the trapped electron effect, the so-called Ohkawa effect. The effects of relativistic mass shift on carrier dynamics and the cyclotron resonance condition were also shown theoretically to be important in determining the ECCD efficiency and profile ${ }^{13,14}$. In tokamaks ECCD can sustain plasma current noninductively in order to reduce the dependence on the ohmic heating coil. More significantly, recent research has shown that optimization of the current profile can bring benefits in plasma stability and confinement ${ }^{15}$ to the extent that economically attractive steady-state operation of tokamak reactors appears to be possible. ECCD is a strong candidate for current profile control because it can be highly localized near the cyclotron resonance in the plasma. This property also makes ECCD useful for stabilizing various magnetohydrodynamic (MHD) instabilities, particularly the neoclassical tearing modes 16,17 , which may limit the achievable plasma pressure.

Quantitative measurements of the properties of ECCD have been made in the DIII-D tokamak ${ }^{18,19}$. These experiments cover a broad range of plasma and wave parameters predicted to be important in influencing the current drive efficiency, like density $\mathrm{n}_{\mathrm{e}}$, temperature $T_{\mathrm{e}}$, normalized minor radius $\rho$, poloidal angle (referring to the location of the current drive on a flux surface), and parallel index of refraction $n_{\|}$. Reference 18 shows that over the entire parameter range tested agreement between the experimental measurement of ECCD and the results of Fokker-Planck calculations of the CQL3D $\operatorname{code}^{20}$ is within experimental uncertainty. This appears to validate the basic ingredients in the physical mechanism of ECCD: the Fisch-Boozer current drive mechanism $^{2}$, the Ohkawa effect ${ }^{1}$, and the relativistic effects ${ }^{13,14}$. 
Although the Fokker-Planck calculations are comprehensive for modeling ECCD, they are time consuming and not convenient for comprehensive modeling of tokamak systems, particularly for time-dependent modeling of operational scenarios. For this purpose, it is desirable to have a simple and relatively accurate method for calculating ECCD efficiency. In the widely used ray tracing code TORAY-GA $21-24$, a simplified model developed by Cohen ${ }^{11}$ is used to evaluate ECCD. This model is based on the Green's function formulation of rf current drive efficiency $25,26,5$. A semi-relativistic response function is used to calculate the current drive. The slowing-down part of the high-velocity collision operator is approximated by an expansion in $\mathrm{T} / \mathrm{mu}^{2}$, where $\mathrm{u}$ is the momentum per unit mass, in order to make the response function for current drive separable in energy and pitch angle. The magnetic well is approximated as a square well in order to obtain an analytic solution. The momentum dependence of the response function thus obtained is judiciously modified, based on Fisch's relativistic theory ${ }^{13}$ for straight-field-line magnetic geometry, to account for relativistic effects.

In the present work fully relativistic electron dynamics are used, starting with Fisch's high-velocity collision model ${ }^{13}$. An approximation based on the Legendre expansion of the pitch angle $\xi=v_{\|} / \mathrm{v}$ is made to the slowing-down part of the collision operator to make the response function separable in energy $w$ and the pitch angle variable $\lambda=\mu / w$, where $\mu$ is the magnetic moment, and $w$ is the particle energy. The resulting mathematical problem may be solved analytically. The solution is shown to be exact in the Lorentz gas limit $\left(Z_{\text {eff }} \gg 1\right.$ ) for general tokamak equilibria, and it recovers Fisch's current drive result ${ }^{13}$ in the limit of large aspect ratio.

The discussion starts with a brief review in Section II of the Green's function formulation for if current drive in general tokamak geometry. We follow the work by Antonsen and $\mathrm{Chu}^{26}$. Their formulation is applicable to all collisionality regimes. A natural local dimensionless current drive efficiency appropriate to general tokamak geometry is introduced. Then it is expressed in terms of the integrals involving the rf-induced flux in velocity space and the response function introduced in the Green's function formulation. In Section III the bounce-averaged equation for the response function in the low collisionality regime is derived. (The low collisionality regime is most relevant for ECCD in present-day and future devices.) In Section IV the relativistic 
high-velocity collision model ${ }^{13}$ is used and the corresponding bounce-averaged equation is solved approximately to obtain a semi-analytic response function for the driven current in general tokamak geometry. In Section $\mathrm{V}$ the theoretical formulation is applied to calculate the ECCD efficiency. The results are compared systematically to those obtained using Cohen's model ${ }^{11}$. A few practical cases using the DIII-D geometry are also presented in which the full ray-tracing is applied. Finally, a summary of the paper is given in Sec. VI. In Appendix A, we present an interpolation formula for the angular part of the response function, which simplifies the numerical evaluation of ECCD efficiency. In Appendix B, we consider the relation between the local dimensionless current drive efficiency defined in this paper and the current drive figure of merit used by experimentalists ${ }^{18,19,28}$. 


\section{GREEN'S-FUNCTION FORMULATION FOR RF CURRENT DRIVE IN TOROIDAL GEOMETRY}

By using the Green's-function formulation to evaluate current drive efficiency, we are assuming that the electron distribution function is close enough to the Maxwellian distribution, i.e., $f \approx f_{\mathrm{M}}$, for the Coulomb collision operator to be linearized, and that the If power density is not too high such that interaction between the waves and electrons can be described by $S_{\text {If }}\left(f_{\mathrm{M}}\right)$, where $S_{\text {If }}$ denotes the rf-induced quasi-linear diffusion operator in velocity space. Ignoring the small cross-field drifts and finite banana width, the perturbed distribution function $f_{1}$ satisfies the linearized Fokker-Planck equation 27 :

$$
\mathrm{v}_{\|} \hat{b} \cdot \nabla f_{1}-C_{\mathrm{e}}^{\ell} f_{1}=S_{\mathrm{rf}}\left(f_{\mathrm{M}}\right)
$$

where $C_{\mathrm{e}}^{\ell}$ denotes the linearized Coulomb collision operator and $f_{1}$ is considered as a function of particle energy $w$, magnetic moment $\mu$, and a poloidal angular variable $\theta_{p}$, at a given flux surface, which could be labeled by the poloidal flux function $\psi$. Within the small gyro-radius expansion scheme which we adopt here, the rf driven current is parallel to the local magnetic field, i.e., $\vec{j}_{\mathrm{rf}}=j_{\|} \vec{B} / B \equiv \dot{j}_{\|} \hat{b}$, with

$$
j_{\|}=-e \int d \Gamma f_{1} v_{\|}
$$

where $d \Gamma$ is the volume element in velocity space. By integrating both sides of Eq. (1) and using the assumption of axisymmetry, it is straightforward to show that $j_{\|}$has a poloidal angular dependence of

$$
j_{\|}=\frac{j_{\|}}{B} B=\gamma(\psi) B
$$


where $\gamma(\psi)$ is a function of $\psi$ only. Hence, $j_{\|} / B$ is independent of the poloidal angle $\theta_{p}$ and can be written as

$$
\frac{j_{\|}}{B}=\frac{\left\langle\dot{j}_{\mid} B\right\rangle}{\left\langle B^{2}\right\rangle}=-e\left\langle\int d \Gamma f_{1} \frac{\mathrm{v}_{\|} B}{\left\langle B^{2}\right\rangle}\right\rangle .
$$

Here the notation (...) denotes the flux-surface average,

$$
\langle\ldots\rangle=\frac{\oint \frac{d \ell_{\mathrm{p}}}{B_{\mathrm{p}}} \ldots}{\oint \frac{d \ell_{\mathrm{p}}}{B_{\mathrm{p}}}},
$$

where $d \ell_{\mathrm{p}}$ is the line element along the poloidal circumference and $B_{\mathrm{p}}$ the poloidal magnetic field.

To evaluate $j_{\|} / B$, it is most convenient to use the Green's-function techniques. Consider the response function for the driven current $\chi$, which satisfies the equation:

$$
-\mathrm{v}_{\|} \hat{b} \cdot \nabla \chi-C_{e}^{\ell+} \chi=\frac{\mathrm{v}_{\|} B}{\left\langle B^{2}\right\rangle}
$$

where $C_{e}^{\ell+}$ is the adjoint collision operator; $C_{\mathrm{e}}^{\ell+} \chi=f_{\mathrm{M}}^{-1} C_{\mathrm{e}}^{\ell}\left(\chi f_{M}\right)$ and

$$
\left\langle\int d \Gamma f C_{\mathrm{e}}^{\ell} g\right\rangle=\left\langle\int d \Gamma\left(C_{\mathrm{e}}^{\ell+} f\right) g\right\rangle
$$

Note that in the equation for the response function (Eq. (6)) we use a different normalization constant from that of Antonsen and $\mathrm{Chu}^{26}$. 
Making use of Eqs. (1)-(7), we express $j_{\|} / B$ in terms of $\chi$ and $S_{\mathrm{rf}}\left(f_{\mathrm{M}}\right)$,

$$
\begin{aligned}
\frac{j_{\|}}{B}=-e\left\langle\int d \Gamma f_{1} \frac{\mathrm{v}_{\|} B}{\left(B^{2}\right\rangle}\right) \\
=-e\left\langle\int d \Gamma f_{1}\left(-\mathrm{v}_{\|} \hat{b} \cdot \nabla \chi-C_{\mathrm{e}}^{\ell+} \chi\right)\right\rangle . \\
=-e\left\langle\int d \Gamma \chi\left(\mathrm{v}_{\|} \hat{b} \cdot \nabla f_{1}-C_{\mathrm{e}}^{\ell} f_{1}\right)\right\rangle \\
=-e\left\langle\int d \Gamma \chi S_{\mathrm{rf}}\left(f_{\mathrm{M}}\right)\right\rangle
\end{aligned}
$$

According to Eq. (1), the absorbed rf power density in the linear regime can be written as

$$
Q=\left\langle\int d \Gamma w S_{\mathrm{rf}}\left(f_{\mathrm{M}}\right)\right\rangle
$$

where $w$ is the particle energy.

Motivated by the introduction of a new dimensionless current drive figure of merit $\zeta$ by experimentalists 28,18 , we define the corresponding local current drive efficiency as

$$
\zeta^{*} \equiv \frac{e^{3}}{\varepsilon_{0}^{2}} \frac{n_{\mathrm{e}}}{T_{\mathrm{e}}} \frac{\left\langle j_{\|}\right\rangle}{2 \pi Q}
$$

where $\varepsilon_{0}$ is the permittivity of free space, $n_{\mathrm{e}}$ and $T_{\mathrm{e}}$ are, respectively, the local electron density and temperature. Using the fact that $\left\langle j_{\|}\right\rangle=\left(j_{\|} / B\right)\langle B\rangle$ and Eqs. (8)-(9), we write $\zeta^{*}$ as

$$
\xi^{*}=-\frac{4}{\ell n \Lambda}\left\langle\frac{B}{B_{\max }}\right\rangle \frac{\left\langle\int d \Gamma \tilde{\chi} S_{\mathrm{rf}}\left(f_{\mathrm{M}}\right)\right\rangle}{\left\langle\int d \Gamma\left(w / m \mathrm{v}_{\mathrm{e}}^{2}\right) S_{\mathrm{rf}}\left(f_{\mathrm{M}}\right)\right\rangle},
$$

where $\ln \Lambda$ is the Coulomb logarithm, $B_{\max }$ is the maximum of $\mathrm{B}$ on the flux surface, and the dimensionless response function is defined as $\tilde{\chi}=v_{\mathrm{e} 0}\left(B_{\max } / v_{\mathrm{e}}\right) \chi$ with

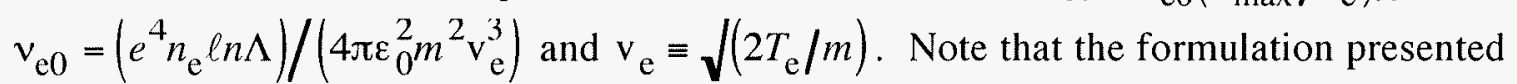
here is applicable for an arbitrary collisionality regime and in general tokamak geometry. With Eq. (11), the problem of evaluating the current drive efficiency is reduced to 
performing integrals involving the wave-induced flux, $S_{\mathrm{rf}}\left(f_{\mathrm{M}}\right)$, and the response function $\chi$. Note that there is no dependence on $S_{\mathrm{rf}}\left(f_{\mathrm{M}}\right)$ in $\chi$. Once determined, it can be used to calculate $\xi *$ for any given $S_{\mathrm{rf}}\left(f_{\mathrm{M}}\right)$. That is one of the advantages of the Green's-function formulation. 


\section{THE BOUNCE-AVERAGED RESPONSE FUNCTION}

To obtain the response function $\chi$, we are required to solve Eq. (6). As it stands, the numerical problem involved is three-dimensional; at a given flux surface, $\chi$ is a function of particle energy $w$, magnetic moment $\mu$, and poloidal angle $\theta_{p}$. To simplify the numerical problem involved, the assumption is often invoked that the effective collisional frequency is much smaller than the bounce frequency such that the trapped electrons are allowed to complete the banana orbits at all energies. This low-collisionality assumption is justified for reactor-grade tokamak plasmas in which the electron temperature is sufficiently high or the velocity of the resonant electrons is much larger than the thermal velocity, so that the influence of collisionality on the current drive efficiency can be neglected. As for the parameter regimes of the present-day experiments, it is generally believed that collisionality corrections on the current drive efficiency would be only significant in the case of strong trapping 29 . Nonetheless, there is no systematic quantitative study of the effects.

In the low-collisionality regime (the "banana" limit), we consider the ratio of the effective collision frequency to the bounce frequency to be a small parameter, $\left(\nu_{\mathrm{e} 0} / \varepsilon\right) / \omega_{b} \ll 1$, where $\varepsilon$ is the inverse aspect ratio. Assume that $\chi$ has an expansion in this small parameter, i.e., $\chi=\chi_{0}+\chi_{1}+\ldots$. According to Eq. (6), the leading order response function $\chi_{0}$ satisfies $\mathrm{v}_{\|} \hat{b} \cdot \nabla \chi_{0}=0$. The equation for $\chi_{1}$ yields the solubility condition,

$$
-\oint \frac{d \ell}{\mathrm{v}_{\|}} C_{\mathrm{e}}^{\ell+} \chi_{0}=\oint d \ell \frac{B}{\left\langle B^{2}\right\rangle},
$$


where $d \ell=\left(B / B_{\mathrm{p}}\right) d \ell_{\mathrm{p}}$. Note that the right-hand side of Eq. (12) is zero for trapped electrons. This implies that $\chi_{0}=0$ for trapped electrons. For passing electrons, Eq. (12) can be written as

$$
\frac{1}{\mathrm{v}}\left(\frac{B}{|\xi|} C_{\mathrm{e}}^{\ell+}\right) \chi_{0}=-\operatorname{sgn}\left(\mathrm{v}_{\|}\right),
$$

by using the definition of $\xi \equiv v_{\|} / v$ and the definition of the flux-surface average (Eq. (5)). In the following, we will limit our discussions to the banana regime, and in referring to $\chi_{0}$ the subscript 0 will be suppressed. 


\section{RESPONSE FUNCTION IN FISCH'S RELATIVISTIC HIGH-VELOCITY COLLISION MODEL}

In this section, we consider Fisch's relativistic high-velocity model ${ }^{13}$ and solve the corresponding bounce-averaged equation for the response function $\chi$ [Eq. (13)]. We will make an approximation on the slowing-down part of the collision operator by keeping only the first term of its Legendre expansion in the pitch angle variable $\xi^{12,30}$. Using this approximation, we obtain an analytic solution for $\chi$ separable in energy and pitch-angle variables in the banana regime.

We use relativistic dynamics to describe the motion of electrons. Let $\vec{u}$ denote the momentum per unit mass, i.e., $\vec{u} \equiv \vec{p} / m=\gamma \vec{v}$ with $\gamma=\sqrt{1}+(u / c)^{2}$. The kinetic energy of a relativistic electron is given by $w=(\gamma-1) m c^{2}$ and the magnetic moment is $\mu=m u_{\perp}^{2} / 2 B$. The collision operator in Fisch's relativistic high-velocity model can be written as

$$
C_{\mathrm{e}}^{\ell} f=\left[v_{\mathrm{ei}}(u)+v_{\mathrm{D}}(u)\right] L f+\frac{1}{u^{2}} \frac{\partial}{\partial u} u^{2} \lambda_{\mathrm{s}}(u) f
$$

Here $L$ is the pitch-angle scattering operator,

$$
L=\frac{1}{2} \frac{\partial}{\partial \xi}\left(1-\xi^{2}\right) \frac{\partial}{\partial \xi} .
$$

The velocity dependent pitch-angle scattering rates due to electron-ion and electronelectron collisions are

$$
v_{\mathrm{ei}}(u)=Z_{\mathrm{eff}} v_{\mathrm{e} 0} \gamma\left(\frac{u_{\mathrm{e}}}{u}\right)^{3},
$$

and

$$
v_{\mathrm{D}}(u)=v_{\mathrm{e} 0} \gamma\left(\frac{u_{\mathrm{e}}}{u}\right)^{3},
$$


where $u_{\mathrm{e}} \equiv \mathrm{v}_{\mathrm{e}}=\sqrt{2 T_{\mathrm{e}} / m}$. The last term on the right-hand side of Eq. (14) represents the slowing-down effects due to electron-electron collisions and

$$
\lambda_{\mathrm{s}}(u)=v_{\mathrm{e} 0} u_{\mathrm{e}} \gamma^{2}\left(\frac{u_{\mathrm{e}}}{u}\right)^{2}
$$

The corresponding adjoint collision operator is

$$
C_{\mathrm{e}}^{\ell+} g=\left[v_{\mathrm{ei}}(u)+v_{\mathrm{D}}(u)\right] L g-\lambda_{\mathrm{s}}(u) \frac{\partial}{\partial u} g
$$

Note that by setting $\gamma \rightarrow 1$ in Eqs. (14)-(19) one recovers the standard non-relativistic high-velocity collision model.

To make progress in obtaining an analytic solution for Eq. (13), we approximate the slowing-down part of the collision operator by keeping only the 1st term of its Legendre expansion in $\xi$ :

$$
\begin{gathered}
C_{\mathrm{e}}^{\ell} f=\left[v_{e \mathrm{i}}(u)+v_{\mathrm{D}}(u)\right] L f+\sum_{\ell} \frac{1}{u^{2}} \frac{\partial}{\partial u} u^{2} \lambda_{\mathrm{s}}(u) f^{(\ell)}\left(u, \theta_{\mathrm{p}}\right) p_{\ell}(\xi) \\
\approx\left[v_{\mathrm{ei}}(u)+v_{\mathrm{D}}(u)\right] L f+\frac{1}{u^{2}} \frac{\partial}{\partial u} u^{2} \lambda_{\mathrm{s}}(u) f^{(1)}\left(u, \theta_{\mathrm{p}}\right) p_{1}(\xi)
\end{gathered},
$$

where $p_{\ell}(x)$ is the Legendre polynomial of order $\ell$ and

$$
f^{(\ell)}\left(u, \theta_{\mathrm{p}}\right)=\frac{2 \ell+1}{2} \int_{-1}^{1} d \xi f\left(u, \xi, \theta_{\mathrm{p}}\right) p_{\ell}(\xi) .
$$

To facilitate calculations in the banana regime, we define a new pitch-angle variable $\lambda$,

$$
\lambda \equiv \frac{\mu B_{\max }}{m u^{2} / 2}=\frac{B_{\max }}{B} \frac{u_{\perp}^{2}}{u^{2}}
$$

which is also a constant of motion. At a given poloidal angle, passing electrons have values of $\lambda$ in the range of $0 \leq \lambda<1$, and for trapped electrons $1 \leq \lambda<B_{\max } / B$. 
Considering $\mathrm{u}$ and $\lambda$ as the two independent variables, the velocity-space volume element is transformed to

$$
d \Gamma=\left(\frac{B_{\max }}{B} \sum_{\operatorname{sgn}\left(\mathrm{u}_{\| 1}\right)} \frac{\pi}{|\xi|} d \lambda\right) u^{2} d u
$$

and $|\xi|=\left(1-\lambda B / B_{\max }\right)^{1 / 2}$, which is considered a function of $\lambda$ and $\theta_{\mathrm{p}}$. We write the bounce averaged pitch-angle scattering operator using

$$
\bar{L} \equiv\left\langle\frac{B}{B_{\max }} \frac{1}{|\xi|} L\right\rangle=2 \frac{\partial}{\partial \lambda} \lambda\langle\mid \xi\rangle \frac{\partial}{\partial \lambda} \text {. }
$$

Substituting Eq. (19) into Eq. (13), using the approximation specified in Eqs. (20)-(21), and rewriting the equation for $\tilde{\chi}$, we obtain

$$
\left(v_{\mathrm{ei}}+v_{\mathrm{D}}\right) \bar{L} \tilde{\chi}-\operatorname{sgn}\left(\mathrm{u}_{\|}\right) \lambda_{\mathrm{s}}(u) \frac{\partial}{\partial u} K(u)=-\operatorname{sgn}\left(\mathrm{u}_{\|}\right) \frac{v_{\mathrm{e} 0}}{\gamma} \frac{u}{u_{e 0}},
$$

with

$$
K(u)=\frac{3}{2}\left\langle\frac{B^{2}}{B_{\max }^{2}} \int_{0}^{1} d \lambda^{\prime}\left[\sigma^{\prime} \tilde{\chi}\left(u, \lambda^{\prime} ; \sigma^{\prime}\right)\right]\right.
$$

and $\sigma^{\prime}=\operatorname{sgn}\left(u_{\|}^{\prime}\right)$ for passing electrons; $\tilde{\chi}=0$ for trapped electrons. It follows that $\tilde{\chi}$ is proportional to $\operatorname{sgn}\left(u_{\|}\right)$for passing electrons, and the fact that $\bar{L} \tilde{\chi}$ is the only term in Eq. (25) having an explicit dependence on $\lambda$ suggests $\tilde{\chi}$ be separable in variables $u$ and $\lambda$. We write the solution of Eq. (25) as

$$
\tilde{\chi}=\operatorname{sgn}\left(u_{\| 1}\right) F(u) H(\lambda)
$$

where the function $H(\lambda)$ satisfies the equation

$$
\bar{L} H(\lambda)=-1,
$$


for $0<\lambda<1$ and vanishes for $\lambda>1$. Eq. (28) and the regular boundary condition at $\lambda=0$ uniquely specify the function $H(\lambda)$ :

$$
H(\lambda) \equiv \frac{\theta(1-\lambda)}{2} \int_{\lambda}^{1} \frac{d \lambda^{\prime}}{\left\langle\left(1-\lambda^{\prime} B / B_{\max }\right)^{1 / 2}\right\rangle}
$$

where $\theta(x)$ is the Heaviside step function such that $\theta(x)=0$ for $x<0$ and $\theta(x)=1$ for $x>0$. Substituting Eqs. (27)-(29) into Eq. (25) and Eq. (26), we find

$$
\begin{aligned}
& K(u)=f_{c} F(u), \\
& \frac{\gamma^{2}}{u^{2}} \frac{\partial}{\partial u} F+\frac{Z_{\mathrm{eff}}+1}{f_{\mathrm{c}}} \frac{\gamma}{u^{3}} F=\left(\frac{1}{f_{c} u_{\mathrm{e}}^{3}}\right) \frac{1}{\gamma} \frac{u}{u_{\mathrm{e}}},
\end{aligned}
$$

with

$$
f_{\mathrm{c}} \equiv \frac{3}{2}\left\langle\frac{B^{2}}{B_{\max }^{2}} \int_{0}^{1} d \lambda H(\lambda)=\frac{3}{4}\left\langle\frac{B^{2}}{B_{\max }^{2}} \int_{0}^{1} \frac{\lambda d \lambda}{\left\langle\left(1-\lambda B / B_{\max }\right)^{1 / 2}\right\rangle} .\right.\right.
$$

The quantity $f_{c}\left(f_{t} \equiv 1-f_{c}\right)$ is the well-known effective circulating (trapped) particle fraction in the neoclassical transport theory 31,32 . The equation for $F$ (Eq. (31)) is readily solved with the boundary condition $F(0)=0$. The solution is

$$
\begin{aligned}
F(u)= & \frac{1}{f_{\mathrm{c}} u_{\mathrm{e}}^{4}}\left(\frac{\gamma+1}{\gamma-1}\right)^{\hat{\rho} / 2} \int_{0} d u^{\prime}\left(\frac{u^{\prime}}{\gamma^{\prime}}\right)^{3}\left(\frac{\gamma^{\prime}-1}{\gamma^{\prime}+1}\right)^{\hat{\rho} / 2} \\
& =\frac{1}{f_{\mathrm{c}}}\left(\frac{u^{4}}{u_{\mathrm{e}}^{4}} \int_{0}^{1} d x x^{\hat{\rho}+3} \frac{1}{\left(1+(u x / c)^{2}\right)^{3 / 2}}\left[\frac{1+\sqrt{1+(u / c)^{2}}}{1+\sqrt{1+(u x / c)^{2}}}\right]^{\hat{\rho}},\right.
\end{aligned}
$$

where

$$
\hat{\rho} \equiv \frac{Z_{\text {eff }}+1}{f_{c}}
$$

Several Remarks on the results obtained so far are in order. We note that the (dimensionless) response function $\tilde{\chi}$ given in Eqs. (27), (29), and (33) is an approximate 
solution for the relativistic high-velocity collision model. For any tokamak equilibrium it is exact in the Lorentz-gas limit $\left(Z_{\text {eff }}>1\right)$, in which the pitch-angle scattering process dominates. We can recover Fisch's results ${ }^{13}$ by considering the limit of large aspect ratio: $\varepsilon \rightarrow 0, B / B_{\max } \rightarrow 1$, and $f_{\mathrm{c}} \rightarrow 1$. Finally, by considering the non-relativistic limit and letting $c \rightarrow \infty$ in Eq. (33), we find

$$
F(u)=\frac{1}{Z_{\text {eff }}+1+4 f_{\mathrm{c}}}\left(\frac{u}{u_{\mathrm{e}}}\right)^{4} .
$$

This result, which was first obtained by Taguchi ${ }^{12}$, indicates that besides the relativistic effects the trapping effects can also alter the canonical $Z_{\text {eff }}$ dependence $\left(1 /\left(Z_{\text {eff }}+5\right)\right)$ of the current drive efficiency in the standard high-velocity collision model. 


\section{ECCD EFFICIENCY}

The theoretical formulation of rf current drive discussed thus far is not specific to any particular wave provided the wave does not significantly alter the electron distribution. In this section, we specialize to the case of ECCD. Following Cohen ${ }^{11}$, we will use a simplified quasi-linear diffusion operator for EC waves. The wave-induced flux $S_{\mathrm{rf}}\left(f_{\mathrm{M}}\right)$ and the response function obtained in Sec. IV are used in Eq. (11) for calculating the ECCD efficiency.

The simplified quasi-linear diffusion operator for EC waves is

$$
S_{r f}(f)=\delta\left(\vec{x}-\vec{x}_{\mathrm{R}}\right) \tilde{\Lambda} D_{0} \delta\left(\omega-k_{\|} \mathrm{v}_{\|}-\ell \frac{\omega_{\mathrm{c}}}{\gamma}\right) \tilde{\Lambda} f
$$

Here $\vec{x}_{\mathrm{R}}$ denotes the spatial location of wave deposition. The differential operator $\tilde{\Lambda}$ in velocity space is given as

$$
\tilde{\Lambda}=\frac{\partial}{\partial w}+\frac{k_{\|}}{\omega} \frac{\partial}{\partial p_{\|}},
$$

where $\omega$ and $k_{\|}$are, respectively, the frequency and the parallel wave number of the EC wave; $w$ and $p_{\| \mid}$are regarded as the two independent velocity-space variables. The local cyclotron frequency at $\vec{x}_{\mathrm{R}}$ is denoted by $\omega_{\mathrm{c}} \equiv e B / m$ and $\ell$ is the cyclotron harmonic number. $D_{0}$ is the quasi-linear diffusion coefficient. We will consider the small gyro-radius limit $\left(k_{\perp} \rho<1\right)$ as has been done in Ref. 11 and approximate $D_{0}$ by

$$
D_{0}=\frac{\pi e^{2}\left|\tilde{E}_{-}\right|^{2}}{2}\left(\frac{k_{\perp} u_{\perp}}{2 \omega_{c}}\right)^{2 \ell-2} \frac{u_{\perp}^{2}}{\gamma^{2}}
$$

where $\tilde{E}_{-}$is the right-hand polarized wave electric field and $k_{\perp}$ is the perpendicular wave number. 
Substituting Eqs. (35)-(36) into Eq. (11) and performing an integration by part in velocity space as well as the flux-surface average, we obtain

$$
\xi^{*}=-\frac{4}{\ell n \Lambda}\left(\frac{B}{B_{\max }}\right) \frac{m u_{\mathrm{e}}^{2} \int d \Gamma D_{0} \delta\left(\omega-k_{\|} \mathrm{v}_{\|}-\ell \frac{\omega_{\mathrm{c}}}{\omega}\right) f_{\mathrm{M}} \tilde{\Lambda} \tilde{\chi}}{\int d \Gamma D_{0} \delta\left(\omega-k_{\|} \mathrm{v}_{\|}-\ell \frac{\omega_{\mathrm{c}}}{\omega}\right) f_{\mathrm{M}}} .
$$

In the process of obtaining Eq. (38), we have also used the fact that $\tilde{\Lambda} w=1$, $f_{\mathrm{M}} \propto \exp \left(-w / T_{\mathrm{e}}\right)$, and $\tilde{\Lambda} f_{\mathrm{M}}=-f_{\mathrm{M}} / T_{\mathrm{e}}$. To evaluate $m u_{e}^{2} \tilde{\Lambda} \tilde{\chi}$, we express $\tilde{\Lambda}$ in terms of partial derivatives with respect to $u$ and $\lambda$. Thus,

$$
\begin{aligned}
m u_{\mathrm{e}}^{2} \tilde{\Lambda} \tilde{\chi} & =\gamma \frac{u_{\mathrm{e}}^{2}}{u} \frac{\partial}{\partial u} \tilde{\chi}+2 \frac{B_{\max }}{B} \frac{u_{\|} u_{\mathrm{e}}^{2}}{\mathrm{u}^{3}}\left(\frac{\gamma u_{\|}}{u}-\frac{n_{\|} u}{c}\right) \frac{\partial}{\partial \lambda} \tilde{\chi} \\
& =\operatorname{sgn}\left(u_{11}\right)\left\{\gamma \frac{u_{\mathrm{e}}^{2}}{u} \frac{d F}{d u} H+2 \frac{B_{\max }}{B} \frac{u_{\|} u_{\mathrm{e}}^{2}}{\mathrm{u}^{3}}\left(\frac{\gamma u_{\|}}{u}-\frac{n_{\|} u}{c}\right) F \frac{d H}{d \lambda}\right\}
\end{aligned},
$$

where $\lambda=\frac{B_{\max } u_{\perp}^{2}}{B u^{2}}$ and $B$ is the magnitude of the local magnetic field at $\vec{x}_{\mathrm{R}}$. Now consider the integration over the velocity-space variables. Note that the volume element in velocity space can be written as $d \Gamma=2 \pi u_{\perp} d u_{\perp} d u_{\|}=2 \pi c^{2} \gamma d \gamma d u_{\|} \quad$ and $\delta\left(\omega-k_{\|} \mathbf{v}_{\|}-\ell \omega_{c} / \gamma\right)=\gamma \delta\left(\gamma \omega-k_{\|} u_{\|}-\ell \omega_{c}\right)$. Substituting Eq. (37) into Eq. (38) and performing the integration over $u_{\| l}$, we find

$$
\xi^{*}=-\frac{4}{\ell n \Lambda}\left(\frac{B}{B_{\max }}\right) \frac{m u_{\mathrm{e}}^{2} \int d \gamma\left(u_{\perp}\right)^{2 \ell} f_{\mathrm{M}} \tilde{\Lambda} \tilde{\chi}}{\int d \gamma\left(u_{\perp}\right)^{2 \ell} f_{\mathrm{M}}}
$$

Note that in Eq. (40) $u_{\|}$and $\gamma$ are related by the cyclotron resonance condition, $\gamma \omega-k_{\|} u_{\|}-\ell \omega_{\mathrm{c}}=0$. Hence, both $u_{\|}$and $u_{\perp}$ are considered as functions of $\gamma$ :

$$
\begin{aligned}
& u_{\|}=\frac{\gamma \omega-\ell \omega_{c}}{k_{\|}}=c\left(\frac{\gamma-y}{n_{\|}}\right), \\
& u_{\perp}^{2}=c^{2}\left(\gamma^{2}-1\right)-u_{\|}^{2}=c^{2}\left[\gamma^{2}-1-\left(\frac{\gamma-y}{n_{\|}}\right)^{2}\right],
\end{aligned}
$$


where $y \equiv \ell \omega_{c} / \omega$ and $n_{\|} \equiv k_{\| \mid} c / \omega$, the parallel index of refraction. The integrations in Eq. (40) are supposed to be taken from $\gamma=1$ to $\infty$. Owing to the cyclotron resonance condition, they could be restricted to the range from $\gamma_{\min }$ to $\gamma_{\max }$. The values of $\gamma_{\min }$ and $\gamma_{\max }$ are determined with given $n_{\|}$and $y$ by setting the left-hand-side of Eq. (42) to zero:

$$
\gamma^{2}-1-\left(\frac{\gamma-y}{n_{\mid}}\right)^{2}=0
$$

Using Eqs. (39)-(43) and the functions $F(u)$ and $H(\lambda)$ obtained in the last section, we can calculate the current drive efficiency $\zeta^{*}$ as defined in Eq. (11). The efficiency $\xi^{*}$ determined here is a function of parallel index of refraction $n_{\|}$, cyclotron harmonic number $\ell$, ratio of the cyclotron frequency to the wave frequency (or $y \equiv \ell \omega_{\mathrm{c}} / \omega$ ), local electron temperature $T_{\mathrm{e}}$, effective ion charge $Z_{\text {eff }}$, as well as the MHD equilibrium properties of the flux surface. The equilibrium information needed are the poloidal angular dependencies of the magnetic field $B\left(\theta_{\mathrm{p}}\right)$, the poloidal magnetic field $B_{\mathrm{p}}\left(\theta_{\mathrm{p}}\right)$, and $d \ell_{\mathrm{p}} / d \theta_{\mathrm{p}}$. Owing to the assumption of small gyro-radius and the simplified quasilinear operator used here, there is no explicit dependence of $\zeta^{*}$ on the polarization state of the EC wave. We note in passing that an interpolation formula which simplifies evaluation of $H(\lambda)$ for general tokamak geometry is presented in Appendix A.

The dimensionless current drive efficiency of Eq. (10) can be compared with experimentally measurable quantities by setting $Q=P / V$ and $I=\left\langle j_{\|}\right\rangle A$, where $V$ and $A$ are, respectively, the volume and the cross sectional area between flux surfaces where the absorption takes place, $\mathrm{P}$ is the total power absorbed, and $\mathrm{I}$ is the total driven toroidal current. Using the approximate relation between $V$ and $A\left(V \approx 2 \pi R_{p} A\right.$, where $R_{p}$ is the major radius of the plasma), one can see $\xi^{*}$ defined in Eq. (10) is directly related to the dimensionless efficiency used by experimentalists $18,19,28$ :

$$
\zeta \equiv \frac{e^{3}}{\varepsilon_{0}^{2}} \frac{n_{\mathrm{e}}}{T_{\mathrm{e}}} \frac{R_{\mathrm{p}} I}{P}
$$

They differ only by geometric factors. In Appendix B, we derive the relevant geometric factors. 
The theoretical formulation developed so far for calculating the ECCD efficiency is not specialized to any specific flux surface geometry. In order to benchmark our results with Cohen's square-well model ${ }^{11}$, we adopt the simple circular model equilibrium with toroidal field $\mathrm{B}=\mathrm{B}_{0} /\left(1+\varepsilon \cos \theta_{\mathrm{p}}\right)$ and poloidal field $\mathrm{B}_{\mathrm{p}}=\mathrm{B}_{\mathrm{p}_{0}} /\left(1+\varepsilon \cos \theta_{\mathrm{p}}\right)$, where $\varepsilon$ is the inverse aspect ratio of the flux surface of interest, $\mathrm{B}_{0}$ and $\mathrm{B}_{\mathrm{p} 0}$ are two constants. For this model equilibrium, both $\zeta^{*}$ and $\zeta$ are functions of the electron temperature $T_{e}$, the parallel wave number $n_{\|}$, the effective ionic charge $Z_{\text {eff }}$, the parameter $y \equiv \ell \omega_{c} / \omega$, the inverse aspect ratio $\varepsilon$, and the poloidal angle $\theta_{p}$ where the absorption takes place. The function $\zeta=\zeta\left(\mathrm{T}_{\mathrm{e}}, \mathrm{n}_{\|}, Z_{\text {eff }}, \mathrm{y}, \varepsilon, \theta_{\mathrm{p}}\right)$ can be plotted as a function of $\mathrm{y}$ with the other arguments held fixed, as shown in some example cases in Fig. 1. The realized efficiency depends on the value of $y$ at which the power is absorbed, and this determination requires tracing of rays from the antenna along with calculation of the power absorbed along the ray. Figure 1 also shows the dimensionless efficiency calculated using the model developed in Ref. 11. For the parameter ranges examined, the relatively small differences between the models shown in Fig. 1 are typical. 


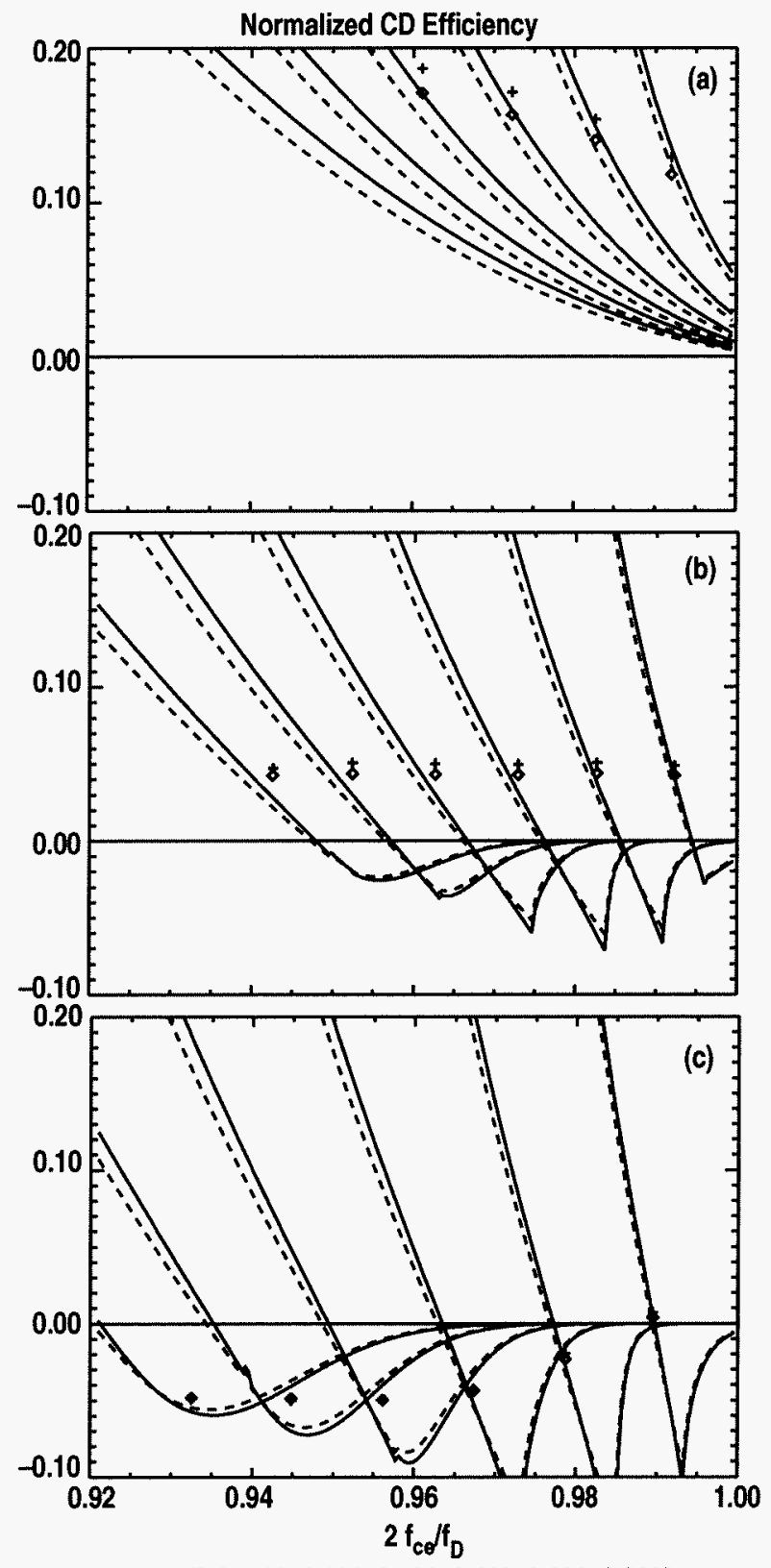

$n \|=[0.700,0.600,0.500,0.400,0.300,0.200]$

Fig. 1. Dimensionless efficiency $\zeta$ defined in Eq. 44, as a function of $y=\ell \omega_{c} / \omega$, evaluated for $\mathrm{n}_{\mathrm{e}}=2 \times 10^{19} \mathrm{~m}^{-3}, \mathrm{~T}_{\mathrm{e}}=2 \mathrm{keV}$, $\mathrm{R}=1.76 \mathrm{~m}, \varepsilon=0.2$ (corresponding to normalized minor radius of 0.5 ), and $Z_{\text {eff }}=1.6$, for (a) $\theta_{p}=165$ degrees (near high-field side of the flux surface), (b) $\theta_{p}=90$ degrees, and (c) $\theta_{p}=15$ degrees. In each case the efficiency is plotted for the specified values of $n_{\|}$. The solid lines represent the results from Eqs. (40-43) and the dotted lines illustrate the results from the model described in Ref. 11 for comparison. 


\section{SUMMARY}

This paper describes the formulation and development of the most complete linear electron cyclotron current drive model for tokamaks. A previously developed model ${ }^{11}$, which has been extensively used in transport modeling and comparison with experiments, uses a weakly relativistic expansion of the Coulomb collision operator. The magnetic geometry is also simplified by use of a square-well model to obtain an analytic solution. While the model has had good success in validation against experiments, the accuracy of the approximations has never been checked against more complete models. In the present work, fully relativistic electron dynamics are used. Recognizing the similarity between this problem and neoclassical transport, an approximation based on Legendre expansion commonly used in the latter is adopted to again arrive at an analytic solution, crucial in terms of speed and accuracy for coupling the current drive model to ray-tracing for time-dependent transport simulations. The analytic solution is shown to be exact in the Lorentz gas limit for general tokamak equilibrium geometry. In comparing with the Cohen model, relatively small differences between the two models have been found in the cases run so far.

Finally, it should be mentioned that the linear ECCD model predicts accurately the scaling of the current drive efficiency for all the relevant plasma parameters. However, it does not account for the presence of an Ohmic electric field and the synergistic contribution of the electric field to ECCD. This effect is most important in present-day experiments in which the current drive has not reached steady-state condition. This physics has been modeled satisfactorily by quasilinear Fokker-Planck codes. For future long-pulse or steady-state tokamaks, the linear ECCD model as described in this paper is expected to provide an accurate model both qualitatively and quantitatively. 


\section{REFERENCES}

[1] T. Ohkawa, National Technical Information Service paper PB2000-108008, NTIS, Springfield, VA 22161.

[2] N.J. Fisch and A.H. Boozer, Physical Review Letters 45, 720 (1980).

[3] V.S. Chan, S.C. Chiu, J.Y. Hsu, and S.K. Wong, Nucl. Fusion 22, 787 (1982).

[4] D.F. Start, J.D. Cordey, and T. Edlington, Plasma Phys. 25, 447 (1983).

[5] M. Taguchi, J. Phys. Soc. Jpn 52, 2035 (1983).

[6] T.M. Antonsen and B. Hui, IEEE Tans. Plasma Sci., PS-12, 118 (1984).

[7] H. Yoshioka, T.M. Antonsen, and E. Ott, Nucl. Fusion 26, 438 (1986).

[8] H. Yoshioka and T.M. Antonsen, Nucl. Fusion 26, 839 (1986).

[9] V.S. Chan, Phys. Fluids 30, 3526 (1987).

[10] G. Giruzzi, Nucl. Fusion 27, 1934 (1987).

[11] R.H. Cohen, Phys. Fluids 30, 2442 (1987), and 31, 421 (1988).

[12] M. Taguchi, Plasma Phys. Cont. Fusion 31, 241 (1989).

[13] N.J. Fisch, Phys. Rev. A24, 3245 (1981).

[14] R.A. Cairns, J. Owen, C.N. Lashmore-Davis, Phys. Fluids 26, 3475 (1983).

[15] A.D. Turnbull, T.S. Taylor, Y.R. Lin-Liu, and H. St. John, Phys. Rev. Lett. 74, 718 (1995).

[16] C.C. Hegna and J.D. Callen, Phys. Plasmas 4, 2940 (1997).

[17] H. Zohm, Phys. Plasmas 4, 3433 (1997).

[18] C.C. Petty, R. Prater, J. Lohr, T. Luce, et al., Nucl. Fusion 42, 1365 (2002). 
[19] C.C. Petty, R. Prater, T.C. Luce, R.A. Ellis, et al., Proceedings, 19th IAEA Fusion Energy Conf., Lyon, France, 2002 (IAEA, Vienna, 2002) paper IAEA-CN94/EX-W-4.

[20] R.W. Harvey and M.G. McCoy, in Proc. of the IAEA Technical Committee Meeting, Montreal, 1992 (IAEA, Vienna, 1993), p.498.

[21] A.H. Kritz, H. Husan, R.C. Goldfinger, and D.B. Batchelor, in Proc. $3^{\text {rd }}$ Joint Varenna-Grenoble Int. Symposium on Heating in Toroidal Plasma (Brussels, Commission of the European Communities, 1982), Vol. II, p709.

[22] K. Matsuda, IEEE Trans. Plasma Sci. 17, 6 (1989).

[23] E. Mazzucato, I. Fidone, and G. Granata, Phys. Fluids 30, 3745 (1987).

[24] K. Matsuda and J.Y. Hsu, Phys. Fluids B3, 414 (1991).

[25] S.P. Hirshman, Phys. Fluids 23, 1238 (1980).

[26] T.M. Antonsen and K.R. Chu, Phys. Fluids 25, 1295 (1982).

[27] T.H. Stix, "Waves in Plasmas" (AIP, New York 1992), p. 522.

[28] T.C. Luce, Y.R. Lin-Liu, R.W. Harvey, et al., Phys. Rev. Lett. 83, 4550 (1999).

[29] Y.R. Lin-Liu, V.S. Chan, F.L. Hinton, and S.K. Wong, Proc. $14^{\text {th }}$ Top. Conf. on RF Power in Plasmas, Oxnard, 2001 (AIP, Melville, N.Y., 2001), p. 438.

[30] F.L. Hinton and Y.B. Kim, Phys. Fluids B5, 3021 (1992).

[31] S.P. Hirshman and D.J. Sigmar, Nucl. Fusion 21, 1079 (1981).

[32] Y.R. Lin-Liu and R.L. Miller, Phys. Plasmas 2, 1666 (1995). 


\section{ACKNOWLEDGMENTS}

This is a report of work supported by the U.S. Department of Energy under Contract Nos. DE-AC03-99ER54463 and DE-FG03-95ER54309. Y.R. Lin-Liu was also partially supported by the National Science Council, Taiwan through Grant No. NSC 90-2112-M-259-008. 


\section{APPENDIX A: AN INTERPOLATION FORMULA FOR $H(\lambda)$}

In this Appendix, we consider an interpolation formula, which simplifies evaluation of the function $H(\lambda)$ defined in Eq. (29) of the main text,

$$
H(\lambda)=\frac{\theta(\lambda-1)}{2} \int_{\lambda}^{1} \frac{d \lambda^{\prime}}{\left\langle\left(1-\lambda^{\prime} h\right)^{1 / 2}\right\rangle},
$$

where $h \equiv B / B_{\max }$. Let's write

$$
\begin{aligned}
(1-\lambda h)^{1 / 2} & =(1-\lambda\langle h\rangle-\lambda(h-\langle h\rangle))^{1 / 2} \\
& =(1-\lambda\langle h\rangle)^{1 / 2}\left[1-\frac{\lambda(h-\langle h\rangle)}{(1-\lambda(h\rangle)}\right]^{1 / 2} \\
& \equiv(1-\lambda\langle h\rangle)^{1 / 2} g
\end{aligned}
$$

In terms of the new variable $s \equiv \lambda(1-\langle h\rangle) /(1-\lambda\langle h\rangle), g$ is given as

$$
g=\left(1-s \frac{h-\langle h\rangle}{1-\langle h\rangle}\right)^{1 / 2}
$$

Note that for $\lambda=0, s=0$ and $\lambda=1, s=1$. Also, $0<h<1$. Hence, both $g$ and $\langle g\rangle$ can be expressed as a power series of $s$ for $s<1$,

$$
\begin{aligned}
\langle g\rangle & =1-s \frac{\langle h-\langle h\rangle)}{(1-\langle h\rangle)}-\frac{1}{8} s^{2} \frac{\left\langle(h-\langle h\rangle)^{2}\right\rangle}{(1-\langle h\rangle)^{2}}+\ldots \\
& =1-\frac{1}{8} s^{2} \frac{\left(\left\langle h^{2}\right\rangle-\langle h\rangle^{2}\right)}{(1-\langle h\rangle)^{2}}+\ldots
\end{aligned}
$$


To carry out the higher order terms in $s$ for evaluating $\langle g\rangle$ may not be particularly fruitful. Here we propose an interpolation formula,

$$
\langle g\rangle \approx\left[1+s^{2}\left(c_{2}\left(1-s^{2}\right)+c_{4} s^{2}\right)\right]^{1 / 2} .
$$

Here the coefficient $c_{2}$ is determined by Eq. (A.4) in the limit of $\mathrm{s}<<1$ and the coefficient $c_{4}$ adjusted to give the exact value at the limit $s=1$. This leads to

$$
\begin{aligned}
& c_{2}=-\frac{1}{4} \frac{\left(\left\langle h^{2}\right\rangle-\langle h\rangle^{2}\right)}{(1-\langle h\rangle)^{2}}, \\
& c_{4}=\frac{\left\langle(1-h)^{1 / 2}\right\rangle^{2}}{(1-\langle h\rangle)}-1 .
\end{aligned}
$$

Three flux-surface averaged quantities are needed for the interpolation formula. They are $\langle h\rangle,\left\langle h^{2}\right\rangle$, and $\left\langle(1-h)^{1 / 2}\right\rangle$.

Expressing $\lambda$ in terms of $s$ and after some straightforward algebra, we find that for $\lambda<1$,

$$
\begin{aligned}
& \frac{d H}{d \lambda}=-\frac{1}{2} \frac{1}{(1-\langle h\rangle)^{1 / 2}} \frac{1}{\left[1+s^{2}\left(c_{2}\left(1-s^{2}\right)+c_{4} s^{2}\right)\right]^{1 / 2}}, \\
& H(\lambda)=\frac{1}{2}(1-\langle h\rangle)^{1 / 2} \int_{s(1-\langle h\rangle+z\langle h\rangle)^{3 / 2}\left[1+z^{2}\left(c_{2}\left(1-z^{2}\right)+c_{4} z^{2}\right)\right]^{1 / 2}}^{d} .
\end{aligned}
$$

For the model circular equilibrium we have considered in the main text, i.e., $B, B_{\mathrm{p}} \propto 1 / 1+\varepsilon \cos \theta_{\mathrm{p}}$ and $d \ell_{\mathrm{p}} \propto d \theta_{\mathrm{p}}$, the flux-surface average of a quantity is given by

$$
\left\langle A\left(\theta_{\mathrm{p}}\right)\right\rangle=\frac{1}{\pi} \int_{0}^{\pi} d \theta_{\mathrm{p}}\left(1+\varepsilon \cos \theta_{\mathrm{p}}\right) A\left(\theta_{\mathrm{p}}\right) .
$$


The three flux-surface averaged quantities required in the interpolation formula can be explicitly expressed in terms of $\varepsilon$ :

$$
\begin{aligned}
& \langle h\rangle=1-\varepsilon, \\
& \left\langle h^{2}\right\rangle=\frac{(1-\varepsilon)^{2}}{\left(1-\varepsilon^{2}\right)^{1 / 2}}, \\
& \left\langle(1-h)^{1 / 2}\right\rangle=\frac{1}{\pi}\left[(1+\varepsilon) \sin ^{-1} \sqrt{\frac{2 \varepsilon}{1+\varepsilon}}+\sqrt{2 \varepsilon(1+\varepsilon)}\right] .
\end{aligned}
$$

With Eqs. (A.11)-(A.13), the coefficients $c_{2}$ and $c_{4}$ can also be expressed as explicit functions of $\varepsilon$. In the limit of $\varepsilon \rightarrow 0, c_{2} \rightarrow-1 / 8$ and $c_{4} \rightarrow 8 / \pi^{2}-1$. By comparison with the numerical results obtained from the exact formulas, we found that the interpolation formulas are quite accurate for the model equilibrium with $0<\varepsilon<0.9$. 


\section{APPENDIX B: GEOMETRIC FACTORS RELATING $\xi^{*}$ AND $\zeta$}

In this Appendix, we derive the geometric factors relating $\zeta^{*}$ defined in Eq. (10) and $\zeta$ introduced in Eq. (44) of the main text.

In general tokamak geometry, the magnetic field is given by

$$
\vec{B}=B_{\phi} \hat{\phi}+\nabla \phi \times \nabla \psi
$$

where $\phi$ is the toroidal angle and $\psi$ is the poloidal flux function. The volume element $\mathrm{V}$ between the flux surfaces labeled by $\psi$ and $\psi+d \psi$ can be written as

$$
V=\oint d \ell_{p} 2 \pi R \frac{d \psi}{|\nabla \psi|}=\left(2 \pi f \frac{d \ell_{p}}{B_{p}}\right) d \psi
$$

The cross sectional area $\mathrm{A}$ between the two flux surfaces is

$$
A=\oint d \ell_{p} \frac{d \psi}{|\nabla \psi|}=\oint d \ell_{p} \frac{d \psi}{R B_{p}}=\left(\left\langle\frac{1}{R}\right\rangle \frac{d \ell_{p}}{B_{p}}\right) d \psi
$$

In writing out Eq. (B.3), we have used the definition of a flux-surface average given in Eq. (5) of the main text. Using the facts that the driven current density is parallel to the local magnetic field, i.e., $\vec{j}=j_{\|} \vec{B} / B$, and $j_{\|} / B$ is a function of $\psi$ only, we write the driven toroidal current $I$ between the two flux surfaces as

$$
I=\oint j_{\phi} d \ell_{p} \frac{d \psi}{|\nabla \psi|}=\left(\frac{j_{\|}}{B} \oint d \ell_{p} \frac{B_{\phi}}{R B_{p}}\right) d \psi=\left(\frac{\left\langle j_{\|}\right\rangle}{\langle B\rangle}\left\langle\frac{B_{\phi}}{R}\right\rangle \frac{d \ell_{p}}{B_{p}}\right) d \psi
$$

Hence,

$$
\begin{aligned}
& I=\left\langle\frac{B_{\phi}}{R}\right\rangle\left(\langle B\rangle\left\langle\frac{1}{R}\right\rangle\right)^{-1}\left\langle j_{\|}\right\rangle A, \\
& V=2 \pi\left\langle\frac{1}{R}\right\rangle^{-1} A .
\end{aligned}
$$


By substituting Eqs. (B.5)-(B.6) and the definition of absorbed power density $Q \equiv P / V$ into Eq. (44) of the main text, we find

$$
\zeta=\frac{R_{p}}{\langle B\rangle}\left\langle\frac{B_{\phi}}{R}\right\rangle \zeta^{*} .
$$

The geometric factors involved pertain to the flux surface of interest. In the limit of large aspect ratio $(\varepsilon \rightarrow 0), \zeta=\zeta^{*}$. 